\title{
Poincaré Symmetry from Heisenberg's Uncertainty Relations
}

\author{
Sibel Başkal ${ }^{1}$ (), Young S. Kim ${ }^{2, *}$ and Marilyn E. Noz ${ }^{3}$ (I) \\ 1 Department of Physics, Middle East Technical University, 06800 Ankara, Turkey; \\ baskal@newton.physics.metu.edu.tr \\ 2 Center for Fundamental Physics, University of Maryland, College Park, MD 20742, USA \\ 3 Department of Radiology, New York University, New York, NY 10016, USA; marilyn.noz@med.nyu.edu \\ * Correspondence: yskim@umd.edu; Tel.: +1-301-937-1306
}

Received: 6 March 2019; Accepted: 18 March 2019; Published: 20 March 2019

\begin{abstract}
It is noted that the single-variable Heisenberg commutation relation contains the symmetry of the $S p(2)$ group which is isomorphic to the Lorentz group applicable to one time-like dimension and two space-like dimensions, known as the $S O(2,1)$ group. According to Paul A. M. Dirac, from the uncertainty commutation relations for two variables, it possible to construct the de Sitter group $S O(3,2)$, namely the Lorentz group applicable to three space-like variables and two time-like variables. By contracting one of the time-like variables in $S O(3,2)$, it is possible to construct the inhomogeneous Lorentz group ISO $(3,1)$ which serves as the fundamental symmetry group for quantum mechanics and quantum field theory in the Lorentz-covariant world. This $I S O(3,1)$ group is commonly known as the Poincaré group.
\end{abstract}

Keywords: Poincaré symmetry from uncertainty relations; one symmetry for quantum mechanics; special relativity

\section{Introduction}

As early as in 1927 [1], Paul A. M. Dirac considered the problem of extending Heisenberg's uncertainty relations to the Lorentz-covariant world. In 1945 [2], he attempted to construct the Lorentz group using the Gaussian wave function. In 1949 [3], Dirac pointed out that the task of constructing relativistic dynamics is to construct a representation of the inhomogeneous Lorentz group. He then wrote down the ten generators of this group and their closed set of commutation relations. This set is known as the Lie algebra of the Poincare group.

In 1963 [4], Dirac considered two coupled harmonic oscillators and constructed an algebra leading to the Lie algebra for the $S O(3,2)$ de Sitter group, which is the Lorentz group applicable to three space dimensions and two time-like variables.

From the mathematical point of view, it is straightforward to contract one of those two time-like dimensions to construct $I S O(3,1)$ or the Poincare group. This is what we present in this paper. However, from the physical point of view, we are deriving the Poincaré symmetry for the Lorentz-covariant quantum world purely from the symmetries of Heisenberg's uncertainty relations.

In Section 2, it is noted that a one-dimensional uncertainty relation contains the symmetry of the $S p(2)$ group in the two-dimensional phase space. It is pointed out that this group, with three generators, is isomorphic to the Lorentz group applicable to two space dimensions and one time variable. We can next consider another set with three additional generators.

In Section 3, we write those Heisenberg uncertainty relations in term of step-up and step-down operators in the oscillator system. It is then possible to consider the two coupled oscillator system with 
the ten generators constructed by Dirac in 1963 [4]. It is gratifying to note that this oscillator system can serve as the basic language for the two-photon system of current interest $[5,6]$.

In Section 4 , we contract one of the time-like variables in $S O(3,2)$ to arrive at the inhomogeneous Lorentz group $I S O(3,1)$ or the Poincaré group. In Section 5 , we give some concluding remarks.

\section{Sp(2) Symmetry for the Single-Variable Uncertainty Relation}

It is known that the symmetry of quantum mechanics and quantum field theory is governed by the Poincaré group [3,7]. The Poincaré group means the inhomogeneous Lorentz group which includes the Lorentz group applicable to the four-dimensional Minkowskian space-time, plus space-time translations [8].

The question is whether this Poincaré symmetry is derivable from Heisenberg's uncertainty relation, which takes the familiar form

$$
\left[x_{i}, p_{j}\right]=i \delta_{i j} \text {. }
$$

There are three commutation relations in this equation. Let us choose one of them, and write it as

$$
[x, p]=i
$$

This commutation relation possesses the symmetry of the Poisson bracket in classical mechanics $[9,10]$. The best way to address this property is to use the Gaussian form for the Wigner function defined in the phase space, which takes the form [11-13]

$$
W(x, p)=\frac{1}{\pi} \exp \left\{-\left(x^{2}+p^{2}\right)\right\} .
$$

This distribution is concentrated in the circular region around the origin. Let us define the circle as

$$
x^{2}+p^{2}=1 .
$$

We can use the area of this circle in the phase space of $x$ and $p$ as the minimum uncertainty. This uncertainty is preserved under rotations in the phase space:

$$
\left(\begin{array}{cc}
\cos \theta & -\sin \theta \\
\sin \theta & \cos \theta
\end{array}\right)\left(\begin{array}{l}
x \\
p
\end{array}\right),
$$

as well as the squeeze of the form

$$
\left(\begin{array}{cc}
e^{\eta} & 0 \\
0 & e^{-\eta}
\end{array}\right)\left(\begin{array}{l}
x \\
p
\end{array}\right) .
$$

The rotation and the squeeze are generated by

$$
J_{2}=-i\left(x \frac{\partial}{\partial p}-p \frac{\partial}{\partial x}\right), \quad K_{1}=-i\left(x \frac{\partial}{\partial x}-p \frac{\partial}{\partial p}\right),
$$

respectively. If we take the commutation relation with these two operators, the result is

$$
\left[J_{2}, K_{1}\right]=-i K_{3},
$$

with

$$
K_{3}=-i\left(x \frac{\partial}{\partial p}+p \frac{\partial}{\partial x}\right) .
$$

Indeed, these three generators form a closed set of commutation relations:

$$
\left[J_{2}, K_{1}\right]=-i K_{3}, \quad\left[J_{2}, K_{3}\right]=i K_{1}, \quad\left[K_{1}, K_{3}\right]=i J_{2} .
$$


This closed set is called the Lie algebra of the $S p(2)$ group, isomorphic to the Lorentz group applicable to two space dimensions and one time dimension.

Let us consider the Minkowskian space of $(x, y, z, t)$. It is possible to write three four-by-four matrices satisfying the Lie algebra of Equation (10). The three four-by-four matrices satisfying this set of commutation relations are:

$$
J_{2}=\left(\begin{array}{llll}
0 & 0 & i & 0 \\
0 & 0 & 0 & 0 \\
i & 0 & 0 & 0 \\
0 & 0 & 0 & 0
\end{array}\right), \quad K_{1}=\left(\begin{array}{cccc}
0 & 0 & 0 & i \\
0 & 0 & 0 & 0 \\
0 & 0 & 0 & 0 \\
i & 0 & 0 & 0
\end{array}\right), \quad K_{3}=\left(\begin{array}{llll}
0 & 0 & 0 & 0 \\
0 & 0 & 0 & 0 \\
0 & 0 & 0 & i \\
0 & 0 & i & 0
\end{array}\right) .
$$

However, these matrices have null second rows and null second columns. Thus, they can generate Lorentz transformations applicable only to the three-dimensional space of $(x, z, t)$, while the $y$ variable remains invariant.

\section{Two-Oscillator System}

To generate Lorentz transformations applicable to the full Minkowskian space, along with $J_{2}, K_{1}$, and $K_{3}$ we need two more Heisenberg commutation relations. Indeed, Paul A. M. Dirac started this program in 1963 [4]. It is possible to write the two uncertainty relations using two harmonic oscillators as

$$
\left[a_{i}, a_{j}^{\dagger}\right]=\delta_{i j}
$$

with

$$
a_{i}=\frac{1}{\sqrt{2}}\left(x_{i}+i p_{i}\right), \quad a_{i}^{\dagger}=\frac{1}{\sqrt{2}}\left(x_{i}-i p_{i}\right)
$$

and

$$
x_{i}=\frac{1}{\sqrt{2}}\left(a_{i}+a_{i}^{\dagger}\right), \quad p_{i}=\frac{i}{\sqrt{2}}\left(a_{i}^{\dagger}-a_{i}\right),
$$

where $i$ and $j$ could be 1 or 2 .

More recently in 1986, this two-oscillator system was considered by Yurke et al. [6] in their study of two-mode interferometers. They considered first

$$
Q_{3}=\frac{i}{2}\left(a_{1}^{\dagger} a_{2}^{\dagger}-a_{1} a_{2}\right)
$$

which leads to the generation of the two-mode coherent state or the squeezed state [5].

Yurke et al. then considered possible interferometers requiring the following two additional operators.

$$
S_{3}=\frac{1}{2}\left(a_{1}^{\dagger} a_{1}+a_{2} a_{2}^{\dagger}\right), \quad K_{3}=\frac{1}{2}\left(a_{1}^{\dagger} a_{2}^{\dagger}+a_{1} a_{2}\right) .
$$

The three Hermitian operators from Equations (15) and (16) satisfy the commutation relations

$$
\left[K_{3}, Q_{3}\right]=-i S_{3}, \quad\left[Q_{3}, S_{3}\right]=i K_{3}, \quad\left[S_{3}, K_{3}\right]=i Q_{3} .
$$

These relations are like those given in Equation (10) for the Lorentz group applicable to two space-like and one time-like dimensions.

In addition, in the same paper [6], Yurke et al. discussed the possibility of constructing interferometers exhibiting the symmetry generated by

$$
L_{1}=\frac{1}{2}\left(a_{1}^{\dagger} a_{2}+a_{2}^{\dagger} a_{1}\right), \quad L_{2}=\frac{1}{2 i}\left(a_{1}^{\dagger} a_{2}-a_{2}^{\dagger} a_{1}\right), \quad L_{3}=\frac{1}{2}\left(a_{1}^{\dagger} a_{1}-a_{2}^{\dagger} a_{2}\right) .
$$


These generators satisfy the closed set of commutation relations

$$
\left[L_{i}, L_{j}\right]=i \epsilon_{i j k} L_{k}
$$

and therefore define a Lie algebra which is the same as that for $S U(2)$ or the three-dimensional rotation group.

We are then led to ask whether it is possible to construct a closed set of commutation relations with the six Hermitian operators from Equations (15), (16) and (18). It is not possible. We must add four additional operators, namely

$$
\begin{array}{ll}
K_{1}=-\frac{1}{4}\left(a_{1}^{\dagger} a_{1}^{\dagger}+a_{1} a_{1}-a_{2}^{\dagger} a_{2}^{\dagger}-a_{2} a_{2}\right), & K_{2}=+\frac{i}{4}\left(a_{1}^{\dagger} a_{1}^{\dagger}-a_{1} a_{1}+a_{2}^{\dagger} a_{2}^{\dagger}-a_{2} a_{2}\right), \\
Q_{1}=-\frac{i}{4}\left(a_{1}^{\dagger} a_{1}^{\dagger}-a_{1} a_{1}-a_{2}^{\dagger} a_{2}^{\dagger}+a_{2} a_{2}\right), & Q_{2}=-\frac{1}{4}\left(a_{1}^{\dagger} a_{1}^{\dagger}+a_{1} a_{1}+a_{2}^{\dagger} a_{2}^{\dagger}+a_{2} a_{2}\right) .
\end{array}
$$

There are now ten operators from Equations (15), (16), (18) and (20). Indeed, these ten operators satisfy the following closed set of commutation relations.

$$
\begin{aligned}
& {\left[L_{i}, L_{j}\right]=i \epsilon_{i j k} L_{k}, \quad\left[L_{i}, K_{j}\right]=i \epsilon_{i j k} K_{k}, \quad\left[L_{i}, Q_{j}\right]=i \epsilon_{i j k} Q_{k},} \\
& {\left[K_{i}, K_{j}\right]=\left[Q_{i}, Q_{j}\right]=-i \epsilon_{i j k} L_{k}, \quad\left[K_{i}, Q_{j}\right]=-i \delta_{i j} S_{3},} \\
& {\left[L_{i}, S_{3}\right]=0, \quad\left[K_{i}, S_{3}\right]=-i Q_{i}, \quad\left[Q_{i}, S_{3}\right]=i K_{i} .}
\end{aligned}
$$

As Dirac noted in 1963 [4], this set is the same as the Lie algebra for the $S O(3,2)$ de Sitter group, with ten generators. This is the Lorentz group applicable to the three-dimensional space with two time variables. This group plays a very important role in space-time symmetries.

In the same paper, Dirac pointed out that this set of commutation relations serves as the Lie algebra for the four-dimensional symplectic group commonly called $S p(4)$, applicable to the systems of two one-dimensional particles, each with a two-dimensional phase space.

For a dynamical system consisting of two pairs of canonical variables $x_{1}, p_{1}$ and $x_{2}$, $p_{2}$, we can use the four-dimensional space with the coordinate variables defined as [14]

$$
\left(x_{1}, p_{1}, x_{2}, p_{2}\right) .
$$

Then the four-by-four transformation matrix $M$ applicable to this four-component vector is canonical if $[15,16]$

$$
M J \tilde{M}=J,
$$

where $\tilde{M}$ is the transpose of the $M$ matrix, with

$$
J=\left(\begin{array}{cccc}
0 & 1 & 0 & 0 \\
-1 & 0 & 0 & 0 \\
0 & 0 & 0 & 1 \\
0 & 0 & -1 & 0
\end{array}\right)
$$

According to this form of the $J$ matrix, the area of the phase space for the $x_{1}$ and $p_{1}$ variables remains invariant, and the story is the same for the phase space of $x_{2}$ and $p_{2}$. 
We can then write the generators of the $S p(4)$ group as

$$
\begin{aligned}
& L_{1}=-\frac{1}{2}\left(\begin{array}{ll}
0 & I \\
I & 0
\end{array}\right) \sigma_{2}, \quad L_{2}=\frac{i}{2}\left(\begin{array}{cc}
0 & -I \\
I & 0
\end{array}\right) I, \quad L_{3}=\frac{1}{2}\left(\begin{array}{cc}
-I & 0 \\
0 & I
\end{array}\right) \sigma_{2}, \\
& S_{3}=\frac{1}{2}\left(\begin{array}{ll}
I & 0 \\
0 & I
\end{array}\right) \sigma_{2},
\end{aligned}
$$

and

$$
\begin{aligned}
& K_{1}=\frac{i}{2}\left(\begin{array}{cc}
I & 0 \\
0 & -I
\end{array}\right) \sigma_{1}, \quad K_{2}=\frac{i}{2}\left(\begin{array}{ll}
I & 0 \\
0 & I
\end{array}\right) \sigma_{3}, \quad K_{3}=-\frac{i}{2}\left(\begin{array}{ll}
0 & I \\
I & 0
\end{array}\right) \sigma_{1}, \\
& Q_{1}=-\frac{i}{2}\left(\begin{array}{cc}
I & 0 \\
0 & -I
\end{array}\right) \sigma_{3}, \quad Q_{2}=\frac{i}{2}\left(\begin{array}{ll}
I & 0 \\
0 & I
\end{array}\right) \sigma_{1}, \quad Q_{3}=\frac{i}{2}\left(\begin{array}{ll}
0 & I \\
I & 0
\end{array}\right) \sigma_{3},
\end{aligned}
$$

where $I$ is the two-by-two identity matrix, while $\sigma_{1}, \sigma_{2}$, and $\sigma_{3}$ are the two-by-two Pauli matrices. The four matrices given in Equation (25) generate rotations, while those of Equation (26) lead to squeezes in the four-dimensional phase space.

As for the difference in methods used in Sections 2 and 3, let us look at the ten four-by-four matrices given in Equations (25) and (26). Among these ten matrices, six of them are diagonal. They are $S_{3}, L_{3}, K_{1}, K_{2}, Q_{1}$, and $Q_{2}$. In the language of two harmonic oscillators, these generators do not mix up the first and second oscillators. There are six of them because each operator has three generators for its own $S p(2)$ symmetry. Let us consider the three generators, $S_{3}, K_{2}$, and $Q_{2}$. For each oscillator, the generators consist of

$$
\sigma_{2}, i \sigma_{1}, \text { and } i \sigma_{3}
$$

These separable generators thus constitute the Lie algebra of $\mathrm{Sp}(2)$ group for the one-oscillator system, which we discussed in Section 2. Hence, the one-oscillator system constitutes a subgroup of the two-oscillator system.

The off-diagonal matrix $L_{2}$ couples the first and second oscillators without changing the overall volume of the four-dimensional phase space. However, to construct the closed set of commutation relations, we need the three additional generators: $L_{1}, K_{3}$, and $Q_{3}$. The commutation relations given in Equation (21) are clearly consequences of Heisenberg's uncertainty relations.

\section{Contraction of $S O(3,2)$ to $\operatorname{ISO}(3,1)$}

Let us next go back to the $S O(3,2)$ contents of this two-oscillator system [4]. There are three space-like coordinates $(x, y, z)$ and two time-like coordinates $s$ and $t$. It is thus possible to construct the five-dimensional space of $(x, y, z, t, s)$, and to consider four-dimensional Minkowskian subspaces consisting of $(x, y, z, t)$ and $(x, y, z, s)$.

As for the $s$ variable, we can make it longer or shorter, according to procedure of group contractions introduced first by Inönü and Wigner [17]. In this five-dimensional space, the boosts along the $x$ direction with respect to the $t$ and $s$ variables are generated by

$$
A_{x}=\left(\begin{array}{ccccc}
0 & 0 & 0 & i & 0 \\
0 & 0 & 0 & 0 & 0 \\
0 & 0 & 0 & 0 & 0 \\
i & 0 & 0 & 0 & 0 \\
0 & 0 & 0 & 0 & 0
\end{array}\right), \quad B_{x}=\left(\begin{array}{lllll}
0 & 0 & 0 & 0 & i \\
0 & 0 & 0 & 0 & 0 \\
0 & 0 & 0 & 0 & 0 \\
0 & 0 & 0 & 0 & 0 \\
i & 0 & 0 & 0 & 0
\end{array}\right),
$$

respectively. The boost generators along the $y$ and $z$ directions take similar forms. 
Let us then introduce the five-by-five contraction matrix [18,19]

$$
C(\epsilon)=\left(\begin{array}{lllll}
1 & 0 & 0 & 0 & 0 \\
0 & 1 & 0 & 0 & 0 \\
0 & 0 & 1 & 0 & 0 \\
0 & 0 & 0 & 1 & 0 \\
0 & 0 & 0 & 0 & \epsilon
\end{array}\right)
$$

This matrix leaves the first four columns and rows invariant, and the four-dimensional Minkowskian sub-space of $(x, y, z, t)$ stays invariant.

As for the boost with respect to the $s$ variable, according to the procedure spelled out in Refs. $[18,19]$, the contracted boost generator becomes

$$
B_{x}^{c}=\lim _{\epsilon \rightarrow \infty} \frac{1}{\epsilon}\left[C^{-1}(\epsilon) B_{x} C(\epsilon)\right]=\left(\begin{array}{ccccc}
0 & 0 & 0 & 0 & i \\
0 & 0 & 0 & 0 & 0 \\
0 & 0 & 0 & 0 & 0 \\
0 & 0 & 0 & 0 & 0 \\
0 & 0 & 0 & 0 & 0
\end{array}\right) .
$$

Likewise, $B_{y}^{c}$ and $B_{z}^{c}$ become

$$
B_{y}^{c}=\left(\begin{array}{ccccc}
0 & 0 & 0 & 0 & 0 \\
0 & 0 & 0 & 0 & i \\
0 & 0 & 0 & 0 & 0 \\
0 & 0 & 0 & 0 & 0 \\
0 & 0 & 0 & 0 & 0
\end{array}\right), \quad B_{z}^{c}=\left(\begin{array}{ccccc}
0 & 0 & 0 & 0 & 0 \\
0 & 0 & 0 & 0 & 0 \\
0 & 0 & 0 & 0 & i \\
0 & 0 & 0 & 0 & 0 \\
0 & 0 & 0 & 0 & 0
\end{array}\right),
$$

respectively.

As for the $t$ direction, the transformation applicable to the $s$ and $t$ variables is a rotation, generated by

$$
B_{t}=\left(\begin{array}{ccccc}
0 & 0 & 0 & 0 & 0 \\
0 & 0 & 0 & 0 & 0 \\
0 & 0 & 0 & 0 & 0 \\
0 & 0 & 0 & 0 & i \\
0 & 0 & 0 & -i & 0
\end{array}\right)
$$

This matrix also becomes contracted to

$$
B_{t}^{c}=\left(\begin{array}{lllll}
0 & 0 & 0 & 0 & 0 \\
0 & 0 & 0 & 0 & 0 \\
0 & 0 & 0 & 0 & 0 \\
0 & 0 & 0 & 0 & i \\
0 & 0 & 0 & 0 & 0
\end{array}\right)
$$

These contraction procedures are illustrated in Figure 1. 


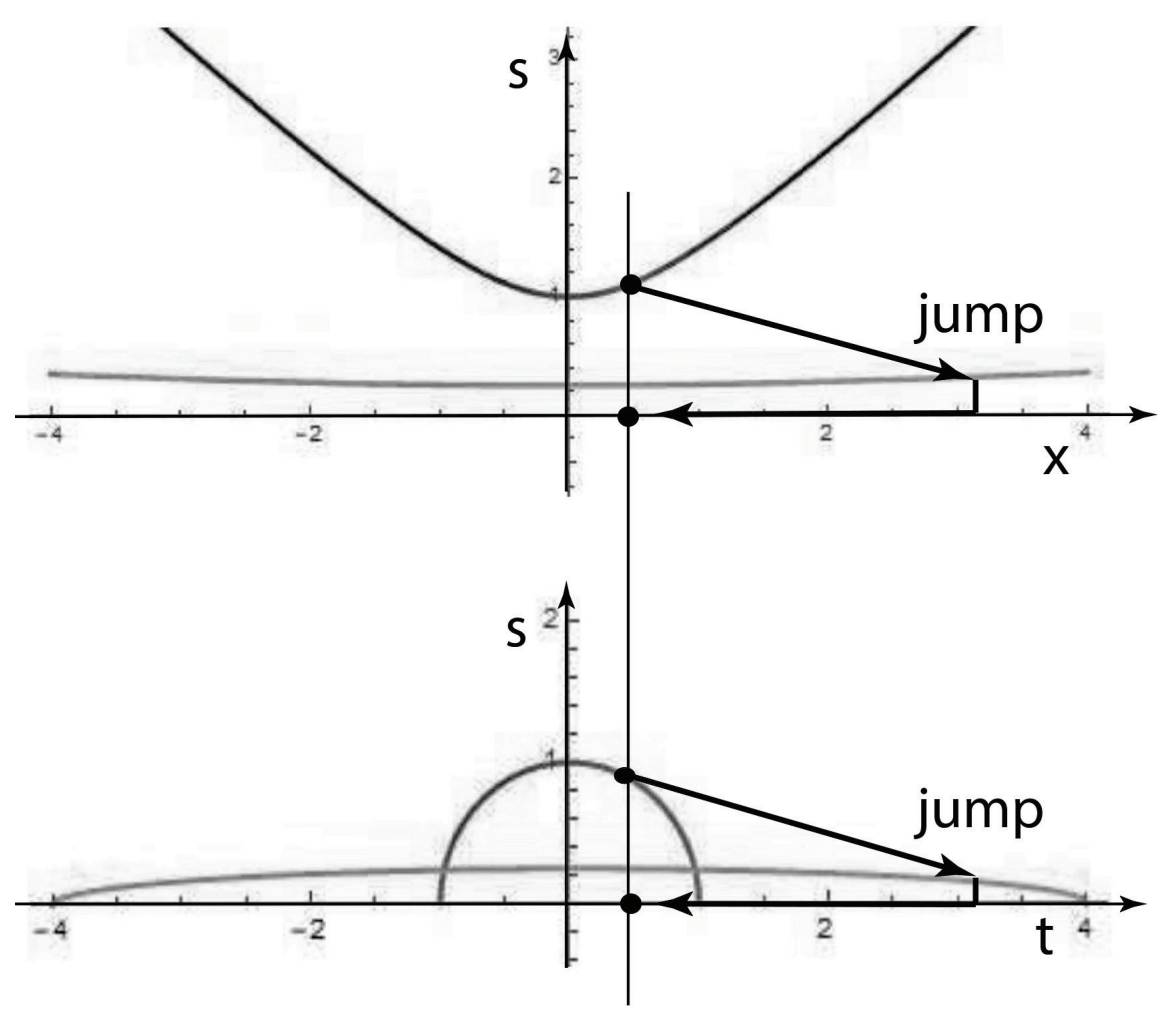

Figure 1. Contraction of the $S O(3,2)$ group to the Poincare group. The time-like $s$ coordinate is contracted with respect to the space-like $x$ variable, and with respect to the time-like variable $t$.

These four contracted generators lead to the five-by-five transformation matrix

$$
\exp \left\{-i\left(a B_{x}^{c}+b B_{y}^{c}+c B_{z}^{c}+d B_{t}^{c}\right)\right\}=\left(\begin{array}{ccccc}
1 & 0 & 0 & 0 & a \\
0 & 1 & 0 & 0 & b \\
0 & 0 & 1 & 0 & c \\
0 & 0 & 0 & 1 & d \\
0 & 0 & 0 & 0 & 1
\end{array}\right)
$$

performing translations:

$$
\left(\begin{array}{lllll}
1 & 0 & 0 & 0 & a \\
0 & 1 & 0 & 0 & b \\
0 & 0 & 1 & 0 & c \\
0 & 0 & 0 & 1 & d \\
0 & 0 & 0 & 0 & 1
\end{array}\right)\left(\begin{array}{c}
x \\
y \\
z \\
t \\
1
\end{array}\right)=\left(\begin{array}{c}
x+a \\
y+b \\
z+c \\
t+d \\
1
\end{array}\right)
$$

This matrix leaves the first four rows and columns invariant. They are for the Lorentz transformation applicable to the Minkowskian space of $(x, y, z, t)$.

In this way, the boosts along the $s$ direction become contracted to the translation. This means the group $S O(3,2)$ derivable from the Heisenberg's uncertainty relations, becomes the inhomogeneous Lorentz group governing the Poincaré symmetry for quantum mechanics and quantum field theory in the Lorentz-covariant world $[4,7]$.

The group contraction has a long history in physics, starting from the 1953 paper by Inönü and Wigner [17]. It starts with a geometrical concept. Our earth is a sphere, but is convenient to consider a flat surface tangent to a given point on the spherical surface of the earth. This approximation is called the contraction of $S O(3)$ to $S E(2)$ or the two-dimensional Euclidean group with one rotational and two translational degrees of freedom. 
This mathematical method was extended to the contraction of the $S O(3,1)$ Lorentz group to the three-dimensional Euclidean group. More recently, Kim and Wigner considered a cylindrical surface tangent to the sphere $[18,19]$ at its equatorial belt. This cylinder has one rotational degree of freedom and one up-down translational degree of freedom. It was shown that the rotation and translation correspond to the helicity and gauge degrees of freedom for massless particles.

Since the Lorentz $S O(3,1)$ is isomorphic to the $S L(2, c)$ group of two-by-two matrices, we can ask whether it is possible to perform the same contraction procedure in the regime of two-by-two matrices. It does not appear possible to represent the ISE(3) (inhomogeneous Euclidean group) with two-by-two matrices. Likewise, there seem to be difficulties in addressing the question of contracting $S O(3,2)$ to $\operatorname{ISO}(3,1)$ within the frame work of the four-by-four matrices of $S p(4)$.

\section{Concluding Remarks}

Special relativity and quantum mechanics served as the major theoretical basis for modern physics for one hundred years. They coexisted in harmony: quantum mechanics augmented by Lorentz covariance when needed. Indeed, there have been attempts in the past to construct a Lorentz-covariant quantum world by augmenting the Lorentz group to the uncertainty relations $[1-3,7,20]$. There are recent papers on this subject [21-23]. There are also papers on group contractions including contractions of the $S O(3,2)$ group [24-26].

It is about time for us to examine whether both of these two great theories can be synthesized. The first step toward this process is to find the common mathematical ground. Before Newton, open orbits (comets) and closed orbits (planets) were treated differently, but Newton produced one differential equation for both. Before Maxwell, electricity and magnetism were different branches of physics. Maxwell's equations synthesized these two branches into one. It is shown in this paper that the group $\operatorname{ISO}(3,1)$ can be derived from the algebra of quantum mechanics.

It is gratifying to note that the Poincaré symmetry is derivable within the system of Heisenberg's uncertainty relations. The procedure included two coupled oscillators resulting in the $\operatorname{SO}(3,2)$ symmetry [4], and the contraction of this $S O(3,2)$ to the inhomogeneous Lorentz group $\operatorname{ISO}(3,1)$.

Author Contributions: Each of the authors participated in developing the material presented in this paper and in writing the manuscript.

Funding: There is no extra funding for this paper.

Conflicts of Interest: The authors declare no conflict of interest.

\section{References}

1. Dirac, P.A.M. The Quantum Theory of the Emission and Absorption of Radiation. Proc. Roy. Soc. 1927, A114, 243-265. [CrossRef]

2. Dirac, P.A.M. Unitary Representations of the Lorentz Group. Proc. Roy. Soc. 1945, A183, 284-295.

3. Dirac, P.A.M. Forms of Relativistic Dynamics. Rev. Mod. Phys. 1949, 21, 392-399. [CrossRef]

4. Dirac, P.A.M. A Remarkable Representation of the $3+2$ de Sitter Group. J. Math. Phys. 1963, 4, 901-909. [CrossRef]

5. Yuen, H.P. Two-photon coherent states of the radiation field. Phys. Rev. A 1976, 13, 2226-2243. [CrossRef]

6. Yurke, B.S.; McCall, B.L.; Klauder, J.R. SU(2) and SU(1,1) interferometers. Phys. Rev. A 1986, 33, 4033-4054.

7. Dirac, P.A.M. The Conditions for a Quantum Field Theory to be Relativistic. Rev. Mod. Phys. 1962, 34, 592-696. [CrossRef]

8. Wigner, E. On unitary representations of the inhomogeneous Lorentz group. Ann. Math. 1939, 40, 149-204. [CrossRef]

9. Arnold, V.I. Mathematical Methods of Classical Mechanics; Springer: Heidelberg, Germany, 1978.

10. Guillemin, V.; Sternberg, S. Symplectic Techniques in Physics; Cambridge University Press: Cambridge, UK, 1984.

11. Han, D.; Kim, Y.S.; Noz, M.E. Linear canonical transformations of coherent and squeezed states in the Wigner phase space. Phys. Rev. A 1988, 37, 807-814. [CrossRef] 
12. Kim, Y.S.; Wigner, E.P. Canonical transformation in quantum mechanics. Am. J. Phys. 1990, 58, $439-447$. [CrossRef]

13. Kim, Y.S.; Noz, M.E. Phase Space Picture of Quantum Mechanics; World Scientific Publishing Company: Singapore, 1991.

14. Han, D.; Kim, Y.S.; Noz, M.E. SO(3,3)-like Symmetries of Coupled Harmonic Oscillators. J. Math. Phys. 1995, 36, 3940-3954. [CrossRef]

15. Abraham, R.; Marsden, J.E. Foundations of Mechanics, 2nd ed.; Benjamin Cummings: Reading, MA, USA, 1978.

16. Goldstein, H. Classical Mechanics, 2nd ed.; Addison-Wesley: Reading, MA, USA, 1980.

17. Inönü, E.; Wigner, E.P. On the Contraction of Groups and their Representations. Proc. Natl. Acad. Sci. USA 1953, 39, 510-524. [CrossRef] [PubMed]

18. Kim, Y.S.; Wigner, E.P. Cylindrical group and massless particles. J. Math. Phys. 1987, 28, 1175-1179. [CrossRef]

19. Kim, Y.S.; Wigner, E.P. Space-time geometry of relativistic-particles. J. Math. Phys. 1990, 31, 55-60. [CrossRef]

20. Yukawa, H. Structure and Mass Spectrum of Elementary Particles. I. General Considerations. Phys. Rev. 1953, 91, 415-416. [CrossRef]

21. Feynman, R.P.; Kislinger, M.; Ravndal, F. Current matrix elements from a relativistic quark model. Phys. Rev. D 1971, 3, 2706-2732. [CrossRef]

22. Loewe, M.; Magnollay, P.; Mukunda, N.; Drechsler, W.; Korny, S.R. Relativistic rotator. I. Quantum observables and constrained Hamiltonian mechanics. Phys. Rev. D 1983, 28, 3020-3031.

23. Bars, I. Relativistic oscillator revisted. Phys. Rev. D 2009, 79, 045009. [CrossRef]

24. Gilmore, R. Lie Groups, Lie Algebras, and Some of Their Applications; Wiley: New York, NY, USA, 1974.

25. Bohm, A.; Loewe, M.; Magnollay, P.; Tarlini, M.; Aldinger, R.R.; Kielanowski, P. New Relativistic Generalization of the Heisenberg Commutation Relations. Pys. Rev. Lett. 1984, 53, 2292-2295. [CrossRef]

26. Bohm, A.; Loewe, M.; Magnollay, P.; Tarlini, M.; Aldinger, R.R.; Biedenharn, L.C.; van Dam, H. Quantum relativistic oscillator. III. Contraction between the algebras of $\mathrm{SO}(3,2)$ and the three-dimensional harmonic oscillator. Phys. Rev. D 1985, 32, 2928-2834. [CrossRef]

(C) 2019 by the authors. Licensee MDPI, Basel, Switzerland. This article is an open access article distributed under the terms and conditions of the Creative Commons Attribution (CC BY) license (http:/ / creativecommons.org/licenses/by/4.0/). 